\title{
A Partial Differential Equation Approach to Image Zoom
}

\author{
Abdelmounim Belahmidi and Frédéric Guichard
}

January 2004

\begin{abstract}
We propose a new model for zooming digital image. This model, driven by a partial differential equation, will balance between linear zooming on homogenous zones to anisotropic diffusion near edges. This allows to combines advantages on linear zoom models and of some non linear ones while leaving out their drawbacks.
\end{abstract}

\section{Introduction}

In this paper, an ideal image is a real function $f$ defined over the plane. That is, we ignore boundary effects and round-off errors. We also consider only one channel - (in practice we will treat the red, blue and green channels separately).

The discretization of $f$ at resolution $\delta x \times \delta y$ is represented mathematically by convolution with the "Dirac comb", i.e., the distribution (see, e.g., [13] for background):

$$
C_{\delta x, \delta y}=\sum_{k, l} \delta_{(k \delta x, l \delta y)}
$$

where $k$ and $l$ are integers. The corresponding discrete image is:

$$
v=C_{\delta x, \delta y} \cdot f
$$

A digital zoom in (or just "zoom" in this paper) is a transformation which takes a discrete image $v$ at some precision $\delta x \times \delta y$ and produce a discrete image $w$ which is an approximation to the discretization of the (ideal) image $f$ at some better precision $\delta x^{\prime} \times \delta y^{\prime}$. That is,

$$
w=C_{\left(\delta x^{\prime}, \delta y^{\prime}\right)} \cdot F
$$

where $F$ is tentative reconstruction of $f$ using only $v$. 


\subsection{Linear Zoom}

A linear zoom is one where this reconstruction is linear and translation-invariant. Linear zooms are by far the most used and studied zooms due to their simplicity and efficiency.

For Linear zooms, $F$ has the form:

$$
F=v * \varphi
$$

for some function $\varphi$ defined on the plane with $\int \varphi(x, y) d x d y=1$. This relation simply indicates that linear zoom differs each other by a specific choice of function $\varphi$ :

$$
w=C_{\left(\delta x^{\prime}, \delta y^{\prime}\right)} \cdot(v * \varphi)
$$

Linear zooms have been quite studied [16]. Among the most famous let us cite the bicubic zoom (or its variant) [10], quadratic zoom [8], b-spline based zoom [17], the zero-padding corresponding to Shannon theory [15], [1] or approximation of it such as e.g. [5] or [4]...

To some (rough) extends, all these zooms differs from a choice of function $\varphi$ and as consequence of some "sharpness" consideration.

In [11], it has been considered the preservation of one dimensional structure. It can be easily seen, that the only linear zoom that fully performs such preservation is the zero-padding [15]. The zero-padding zoom introduces on another side some ringing effects along boundaries due to the cut-off in frequencies (high frequencies are set to 0 ). All others linear zooms introduce non zero values in high frequencies following a periodicity pattern (see e.g. figure 2).

\subsection{Linearly Invertible (linear or non linear) Zoom}

When discussing non-linear techniques for zooming images, the field of possible of non linear zoom is obviously bigger than the linear one. However, an interesting restriction has been introduced in [9]. The authors propose to only consider non linear zoom whose inverse operation (zoom out to the original image) can be written as a linear zoom. With such an assumption the zoom operator should satisfy a similar expression than equation 1 , but an expression which is open to a little more flexibility.

$$
v=C_{\delta x, \delta y}(S * w)
$$

Or, in the Fourier domain:

$$
\tilde{v}(m, n)=\sum_{k, l} \tilde{w}(m+k M, n+l N) \tilde{S}(m+k M, n+l N)
$$

In other words, Fourier coefficients of the zoomed image $w$ and original image $v$ are linked by a linear equation. Zoom methods, satisfying this constraint, differ in a (linear or non linear) choice of distributing energy for each coefficient $\tilde{v}(m, n)$ into the coefficients $\tilde{w}(m+k M, n+l N)$. Given an image $v$ and a choice of inverse zoom function $S$, the set of image satisfying the equation 2 is a linear subspace. In the following we will call it $\mathcal{W}_{v, S}$. 
Among several examples, let us discuss two of such zoom: Using Bayesian approach Schultz et Stevenson [14] have proposed to choose as $v$ the function that minimizes the following energy:

$$
\min \left\{\sum_{i, j} \sum_{k=1}^{4} \rho\left(D^{2} v\left(\nu_{k}, \nu_{k}\right)_{i, j}\right)\right\}
$$

under the constraint given by equation 2. $D^{2} v\left(\nu_{k}, \nu_{k}\right)_{i, j}$ is a finite difference approximation of the seconde derivatives computed in one of the four directions, $\rho(x)$ is equal to $x^{2}$ for $|x|<T$ and extended linearly beyond $|x|=T$ such that $\rho$ is smooth and convex. An interesting property of this method lies in the threshold parameter $T$ which is used to act differently on smooth regions and on non-smooth regions. However, due to the second derivative term within the energy, it is difficult to draw properties of the model. In addition, the energy is quite difficult to minimize due to instabilities of the PDE derived from the gradient descent.

In a quite similar idea, Guichard et al. [9] proposed to choose among $\mathcal{W}_{v, S}$ the image that has the smallest total variation: $E(w)=\int|D w|$. Solutions of the problem are not unique. However, the authors show that all solutions can be deduced from each others by some specific transformations. So that minimization can be performed easily by a gradient descent from any linearly zoomed version of the original image to one of the solutions. This zoom shares advantages and disadvantages of the total variation minimization: as total variation, it allows discontinuities and therefore strong sharpness and it preserves one dimensional structures, but it over-smoothes homogeneous regions.

To summarize, these two methods consist in minimizing an energy under the constraint 2. That is, choosing among possible images $\mathcal{W}_{v, S}$, the image that has the smallest energy. As consequence, choosing a zoom method boils down with such approach to the choice of a good energy - which is somehow one of the main issue of variational-based image processing.

\section{A new zoom method}

Somehow we wish to choose, among the linearly invertible zoom, one that benefits the advantages of all the preceding methods. A good zoom should be as good as linear ones on homogeneous region and as good as e.g. Total variation based zoom on edges.

\subsection{Our model}

Let $v$ be the image to expand and $u_{0}$ be an initial reconstruction of $v$. We have $v=C_{\delta x, \delta y} \cdot u_{0}$ and we will discuss later a specific choice of reconstruction.

We denote by $P$ the orthogonal projection on to the space $\mathcal{W}_{v, S}$. The zoomed $\left(d \times d\right.$ times) version of $v$ is the image $w=C_{\delta x / d, \delta y / d} u(\cdot, T)$, where $u(\cdot, T)$ is 
the solution at scale $T$ ( $T$ fixed later) of the following equation:

$$
\begin{gathered}
\frac{\partial u}{\partial t}=|D u| D^{2} u(\xi, \xi)+g(|D u|) D^{2} u(\eta, \eta) \\
-\left(P u-P u_{0}\right),
\end{gathered}
$$

with initial condition given by $u_{0}$ : that is $u(0, \cdot)=u_{0}(\cdot)$, and where $\xi$ and $\eta$ are unit vectors respectively orthogonal and in the direction of the gradient $D u$ of $u(t, \cdot)$. That is:

$$
\xi=D u^{\perp} /|D u|, \quad \eta=D u /|D u| .
$$

Terms of the PDE can be interpreted as follow: the first one diffuses $u$ only in the orthogonal direction of the gradient. The second term diffuses $u$ only in the direction of the gradient. The last term forces $u$ to stay near the sub-space: $W_{v, S}$. The function $g$ is to be chosen with values between 0 and 1 . It somehow balances the two diffusion terms. When $g=1$, the two diffusion terms have an equal weight and as a consequence the two first term can be combined into a linear and isotrop diffusion $(\partial u / \partial t=\Delta u)$. When $g=0$, only the diffusion in the orthogonal direction of the gradient opers. A deeper analysis of the two first terms of the PDE follows in the next subsection.

For the choice of $g$ we use the Malik and Perona edge-stopping function $g(s)=1 /\left(1+(s / \lambda)^{2}\right)$. In fact one of the first ideas to process separately the homogenous areas and edges, is coming from Malik and Perona [12] and consists to evolve the image with the anisotropic diffusion : $\partial u / \partial t=\operatorname{div}(g(|D u|) D u)$. In particular for large magnitude of the gradient the diffusion is stopped and edges are conserved.

With such choice of function $g$, assuming $u_{0}$ is continuous, our model has a unique solution $u(t, \cdot)$ in the viscosity framework [7]. Complete proof can be found in [3]. Let us also note that any discretization of the PDE satisfying asymptotic behavior and monotony is consistent with the equation. This roughly means that the equation can be numerically solved by classic schemes.

\subsection{Major Properties of our Model}

Behavior of the PDE is driven by the function $g$.

On homogeneous zones of $u, g$ will have values near 1 . In that case, the two first terms of the PDE will combined into the laplacian of $u$, yielding an isotropic linear diffusion. Since the projection term is a linear term. The equation performs a linear reconstruction which could be compared to a linear zoom.

On edges, $g$ will have values near 0 . In that case, the second term of the PDE vanishes, leaving the anisotropic diffusion of the first term. This term is nothing but the mean curvature motion operator (see [2]). It smoothes all level lines of the evolving image. This will force edges geometry to be smoothed without any effect on edges sharpness. First term can be also be interpreted as a 1D diffusion: along the orthogonal direction of the gradient. To some extend it performs a 1D linear zooming, where the direction of the zoom is locally set to the tangent of the edges. 
In fact here is the main property of the PDE: when the image is homogeneous it performs a linear zoom and when the image is locally 1D oriented (edge) it performs a 1D linear zoom...

\subsubsection{Choice of diffusion time}

Choice of the diffusion time $T$ is linked to the zooming factor $d$. We wish to smooth evolving image $u$ until details that are too small to exist in the original image $v$ disappear. As we have seen, on homogenous zones the PDE behaves as the heat equation whose solution is the convolution by a gaussian kernel. Evolving an image $u_{0}$ with the at scale $t$ heat equation is equivalent to compute the convolution with a gaussian kernel whose mean deviation is $\sqrt{2 t}$. So that, for a zoom of factor $d$, we wish to set this mean deviation to $\sqrt{2} d$ (diagonal of an initial pixel expressed in unit of pixels of the zoom image). This simply yields $T=d^{2}$.

\subsection{Discretization of the PDE}

To discretize the PDE, we do not start with $u_{0}$ defined all over the plan, but with a discrete $u_{0}$ having resolution of the zoomed image. Our scheme is the following : We begin with an image $u_{0}$ satisfying $P u_{0}=u_{0}$, e.g. the interpolation with duplication (of factor $d$ ). We then iterate with the explicit scheme :

$$
\begin{gathered}
\frac{u_{i j}^{n+1}-u_{i j}^{n}}{\delta t}=D^{2} u^{n}(\xi, \xi)_{i j}+g\left(\left|D u^{n}\right|\right) D^{2} u^{n}(\eta, \eta)_{i j} \\
-P\left(u^{n}-u_{0}\right)_{i j} .
\end{gathered}
$$

Where $D^{2} u^{n}(\xi, \xi)_{i j}$ and $D^{2} u^{n}(\eta, \eta)_{i j}$ are computed with $3 \times 3$ stentil using the scheme proposed in [6]. The Neumann type boundary is also used. For the experiments, we choose $S$ being the caracteristic function of a pixel of image $v$. So that the reduction term $P$ on the image subspace $\mathcal{W}_{v, S}$ can be written as

$$
P u(x)=\sum_{i, j} \mathbb{I}_{\Omega_{i, j}}(x) d^{-2} \int_{\Omega_{i, j}} u(y) d y,
$$

where $\Omega_{i, j}$ is the corresponding square $d \times d$ of the expansion of pixel $(i, j), \mathbb{I}_{\Omega_{i, j}}$ is its caracteristic function of $\Omega_{i, j}$.

\section{Experiments}

Figure 1 illustrates different magnifications of factor 4 of part of the famous Lenna image. The zoom by duplication a quantization effect appears in the homogenous areas and "stair-case" effects are visible. The cubic B-spline interpolation smoothes the expended image and edges. The zoom based on total variation leaves edges sharp but the homogenous areas tends to become

piecewice constants. Our zoom clearly avoid all of these drawbacks. In Figure 
2 we display the modulus of images spectra of figure 1. Remark how well, compare to others, the frequency spectrum is well extrapolated by our model. At last, Figure 3, we compare our model and b-spline interpolation on a textured image.
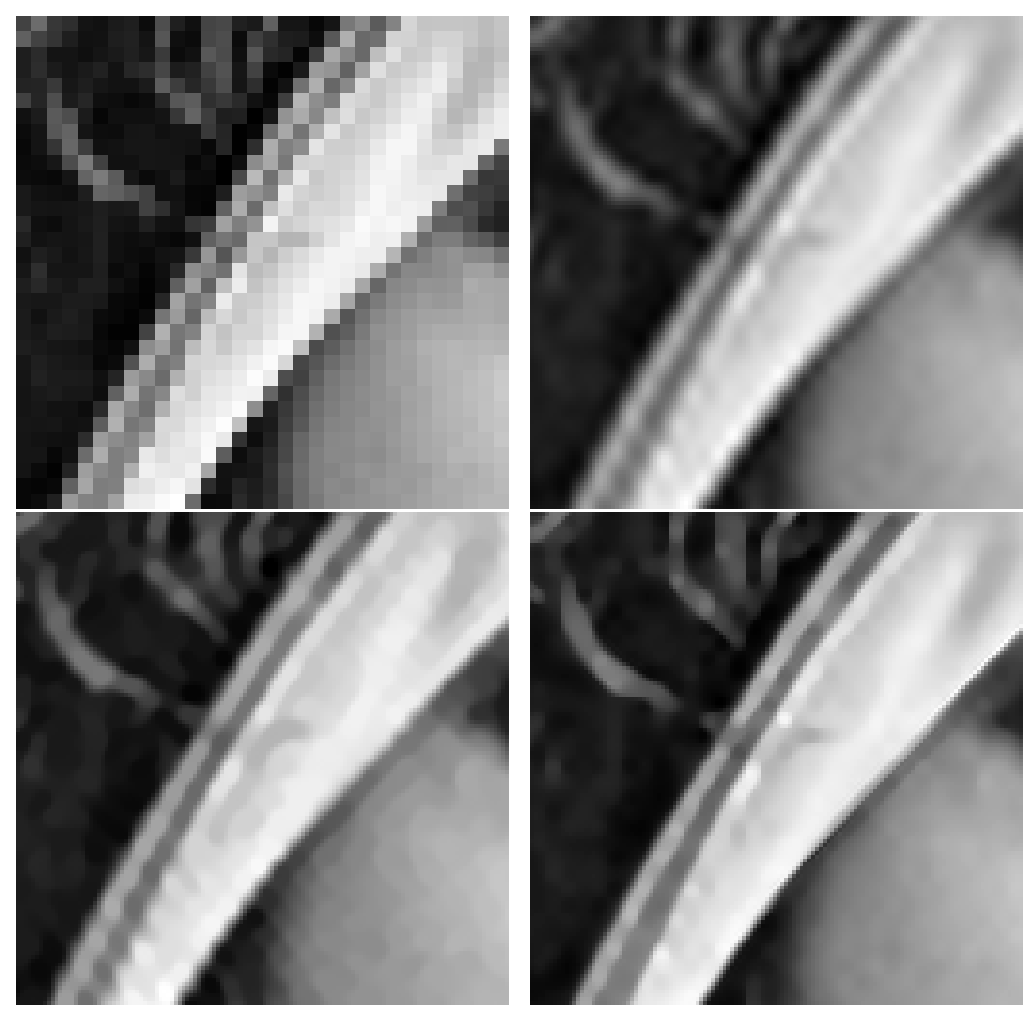

Figure 1: Part of Lenna image, Up-left : zoomed $\mathrm{x} 4$ by duplication, Up-right : by cubic B-spline interpolation, Down-left : by the minimisation of the total variation of the image, Down-right : by our model. 

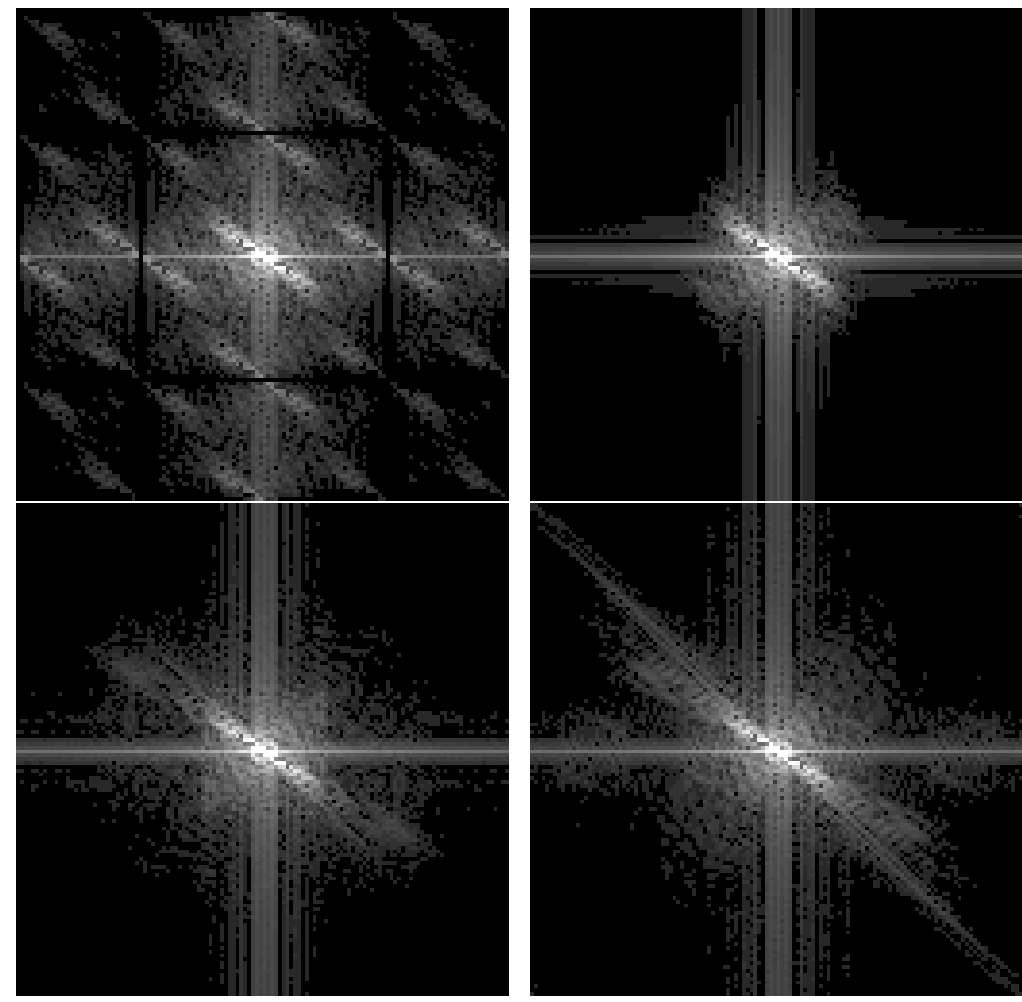

Figure 2: Illustration of modulus of spectra of the images in figure 1. We clearly see, for the two linear zooms a duplication or a amplitude falloff for high frequencies. TV based zoom performs an extrapolation preserving main directions. Our model performs this even further.

\section{Conclusion}

We have introduced a zooming whose inverse is linear and that benefits advantages of linear zooms on homogenous areas, and of total variation zooms on image edges. We observe experimentaly that our method - preserves one dimensional structures (and therefore avoid stair-case effect), - outperforms linear zooms as well as total variation based zoom in term of Fourier spectrum extrapolation, - compares advantageously with classic zooms.

Acknoledgment. The first author would like to thank A. Chambolle for all fruitful discussions. 


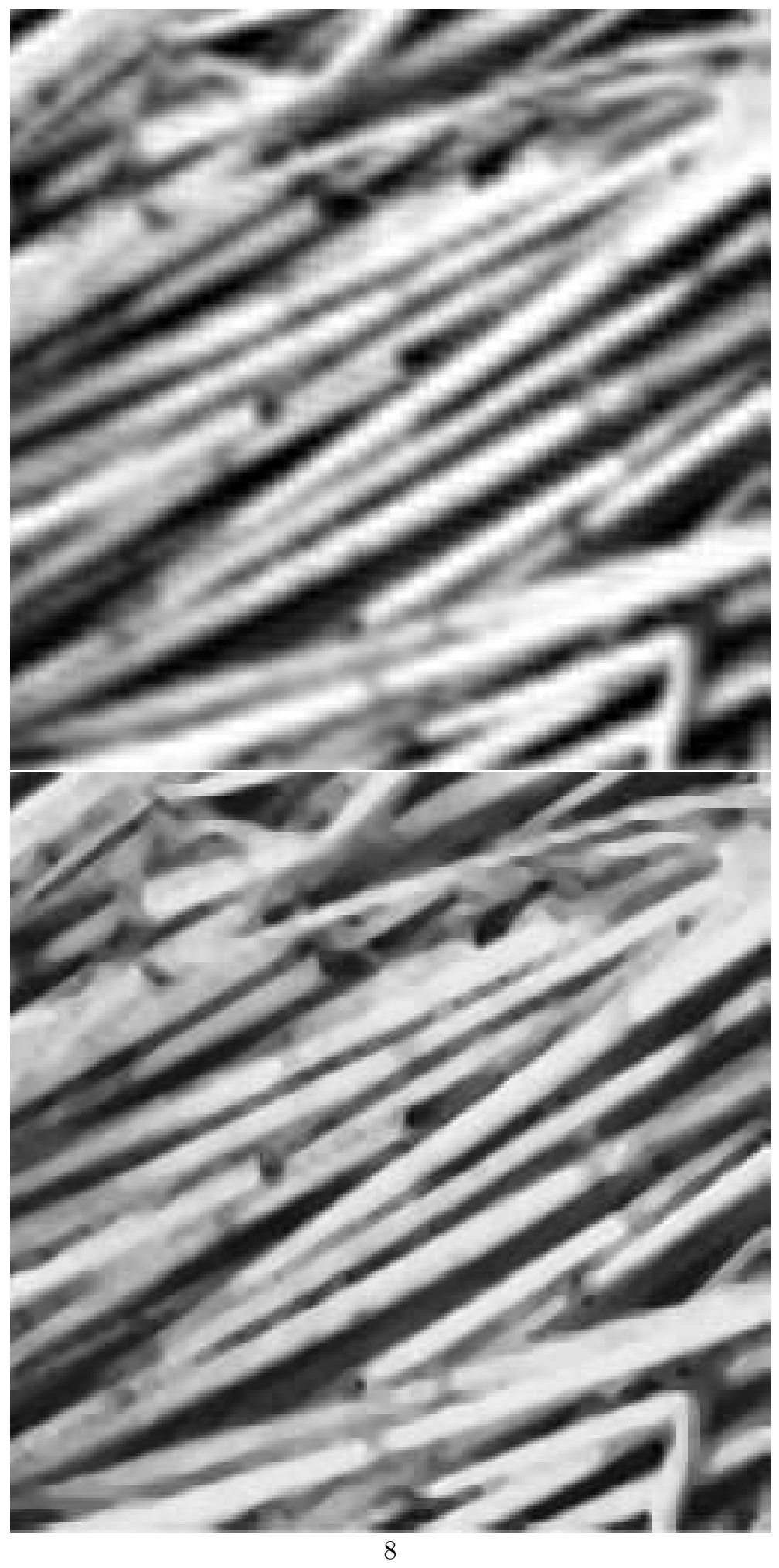

Figure 3: Texture image zoomed $\times 4 \mathrm{Up}$ : by cubic B-spline interpolation, Down : by our model. 


\section{References}

[1] J. Agbinya. Interpolation using the discrete cosine transform. Electronics Letters, U. K., 28(20):1927-1928, 1992.

[2] L. Alvares, F. Guichard, P. L. Lions, and J. M. Morel. Axioms and fundamental equations of image processing. Arch. Rational Mech. Anal., 123(3):199-257, 1993.

[3] A. Belahmidi. PDEs applied to image restoration and image zooming. $\mathrm{PhD}$ thesis, CEREMADE, Université de Paris XI Dauphine, 2003.

[4] T. Blu, P. Thévenaz, and M. Unser. Moms: maximal order interpolation of minimal support. IEEE Trans. Image Proc., 10(7):1069-1080, 2001.

[5] F. Brouaye. Sampling and wavelets : a new approach to the zero-padding method. Traitement du signal. France, 2(9):193-200, 1992.

[6] T. Cohignac, F. Eve, F. Guichard, C. Lopez, and J.M. Morel. Affine morphological scale-space : Numerical analysis of its fundamental equation. Technical Report 9254, CEREMADE, 1992.

[7] M. G. Crandall, H. Ishii, and P. L. Lions. User's guide to viscosity solutions of second order pertial differential equations. Bull. Amer. Soc., 27:1-67, 1992.

[8] N. A. Dodgson. Quadratic interpolation for image resampling. IEEE Transactions on image processing, 6(9):1322-1326, Sept. 1997.

[9] F. Guichard and F. Malgouyres. Total variation based interpolation. EUSIPCO, III, pages 1741-1744, 1998.

[10] R. G. Keys. Cubic convolution interpolation for digital image processing. IEEE Transaction on Acoustics, Speech ans Signal Processing, 29:1153-1160, 1981.

[11] F. Malgouyres and F. Guichard. Edge direction preserving image zooming: a mathematical and numerical analysis. SIAM J. Num. Anal., 39(1):1-37, 2002.

[12] J. Malik and P. Perona. A scale space and edge detection using anitropique diffusion. IEEE Comp. Soc. Workshop on Comp. Vision, Miami, pages 16-22, 1987.

[13] W. Rudin. Functional analysis. McGraw-Hill, New York, 1991.

[14] R. R. Schultz and R. L. Stevenson. A bayesian approach to image expansion for improved definition. IEEE Transactions on Image Processing, 3(3):233-242, 1994.

[15] C.E. Shannon. Communication in the presence of noise. Proc. I.R.E., 37:10-21, 1949.

[16] T.Blu and M.Unser. Quantitative fourier analysis of approximation techniques: Part i -interpolators and projectors, part ii-wavelets. IEEE Trans. Signal Process., 47(10):2783-2806, 1999.

[17] M. Unser, A. Aldroubi, and M. Eden. Fast b-spline transforms for continuous image representation and interpolation. IEEE Trans. on pattern Analysis and machine intelligence, 13(3):277-285, 1991. 